\title{
MONOTONE OPERATOR FUNCTIONS ON ARBITRARY SETS
}

\author{
WILLIAM F. DONOGHUE, JR.
}

\begin{abstract}
We give a new proof of a result of Chandler which shows that a monotone operator function defined on a set $J$ admits an analytic continuation to the upper and lower half-planes, and that this continuation is a Pick function, real and regular on the convex hull of $J$.
\end{abstract}

Let $J$ be a subset of the real axis and $f(x)$ a real-valued function defined on $J$. If $H$ is a selfadjoint operator on Hilbert space having a spectrum contained in $J$, the operator $f(H)$ is defined by the usual operational calculus:

$$
f(H)=\int f(\lambda) d E_{\lambda}
$$

where $E_{\lambda}$ is the resolution of the identity corresponding to $H$. Here $f(H)$ is defined only if $f(x)$ is subject to certain mild measurability conditions. However, in the special case when the Hilbert space is finite dimensional, no measurability conditions need be imposed for $f(H)$ to make sense, and indeed, if $H$ is represented by a diagonal matrix with eigenvalues $\lambda_{i}$, then $f(H)$ is represented by another diagonal matrix with eigenvalues $f\left(\lambda_{i}\right)$.

The function $f(x)$ is said to be a monotone matrix function of order $n$ if, for any $n$-dimensional Hilbert space and any pair of selfadjoint operators $A$ and $B$ on that space having their spectra in $J$, the operator inequality $A<B$ implies $f(A)<f(B)$. The function $f(x)$ is called a monotone operator function if a similar assertion holds for infinite dimensional Hilbert space.

In the special case when the set $J$ is an open interval of the real axis the theory of monotone operator functions and monotone matrix functions is more or less complete. A theorem of Bendat and Sherman [1] asserts that the monotone operator functions defined on the interval $J$ are exactly those functions that are monotone matrix functions of all orders on the interval. A well-known theorem of Loewner [6] then guarantees that these functions are precisely those real functions on the interval $J$ which admit an analytic continuation throughout the upper half-plane having a positive imaginary part in that half-plane. The functions are also continuable by reflection across $J$ to the lower half-plane. Since every Pick function, (i.e., one analytic in the upper half-plane with positive imaginary part) admits a unique canonical representation of the form

$$
f(z)=\alpha z+\beta+\int\left[\frac{1}{\lambda-z}-\frac{\lambda}{\lambda^{2}+1}\right] d \mu(\lambda)
$$

Received by the editors January 10, 1979.

AMS (MOS) subject classifications (1970). Primary 47A60, 47B15; Secondary 30A14. 
where $\alpha \geqslant 0, \beta$ is real and $d \mu$ is a positive measure for which the function $\left(\lambda^{2}+1\right)^{-1}$ is integrable, all monotone operator functions on $J$ have such a representation. It is easy to verify that a function of the form (1) is a monotone operator on $J$ if and only if the corresponding measure puts no mass in the open interval $J$.

In recent years there has been considerable interest in the study of monotone operator functions defined on $J$ when $J$ is an arbitrary open set. Let $\left(a_{i}, b_{i}\right)$ be the constituent intervals of $J$. If $f(x)$ is a monotone operator function on $J$ it is surely a monotone operator function on each of the constituent intervals and so admits, from each such interval, an analytic continuation to the upper half-plane. It is tempting to suppose that the continuation so obtained is independent of the interval in question, i.e., that $f(x)$ is of the form (1) where the measure $d \mu$ puts no mass in the open set $J$. That this is in fact the case is one of the consequences of work of Rosenblum and Rovnyak [7]. Their result has been extended by Chandler [2] who has shown that in fact the measure $d \mu$ puts no mass in the convex hull of $J$. His results depend on the deep investigations of Rosenblum and Rovnyak as well as results of Šmul'jan [8] which in turn depend on studies of Davis ([3], [4]). We give here a simpler proof of Chandler's theorem in the spirit of the original work of Loewner. Here, if $J$ is a set, $c(J)$ is its convex hull.

Chandler's Theorem. A monotone operator function $f(x)$ defined on the open set $J$ is of the form (1) where the measure du puts no mass in the open interval $c(J)$. The function is therefore the restriction to $J$ of a monotone operator function on $c(J)$.

Proof. We first establish the theorem under the additional hypothesis that $J$ is a bounded set. Let $\left(a^{\prime}, b^{\prime}\right)$ and $\left(a^{\prime \prime}, b^{\prime \prime}\right)$ be two constituent intervals of $J$; we may suppose that $\left(a^{\prime}, b^{\prime}\right)$ is the one on the left. Select a dense sequence $\left\{x_{k}^{\prime}\right\}$ in $\left(a^{\prime}, b^{\prime}\right)$ and another sequence $\left\{x_{k}^{\prime \prime}\right\}$ dense in $\left(a^{\prime \prime}, b^{\prime \prime}\right)$. Let $\varepsilon_{n}$ be a sequence of positive numbers converging rapidly to 0 and let $\Psi(x)$ be a monotone operator function defined on $c(J)$ which is not rational. It is easy to find such a function, since any function of the form (1) where the measure has no mass in the interval $c(J)$ and is not a finite collection of point masses will do.

For each positive integer $\boldsymbol{n}$ consider the set

$$
S_{n}=\left\{x_{1}^{\prime}, x_{2}^{\prime}, x_{3}^{\prime}, \ldots, x_{n}^{\prime}, x_{1}^{\prime \prime}, x_{2}^{\prime \prime}, x_{3}^{\prime \prime}, \ldots, x_{n}^{\prime \prime}\right\}
$$

consisting of $2 n$ points of $J$ and let $\varphi_{n}(z)$ be a rational function of degree at most $2 n$ satisfying the $2 n$ equations

$$
\varphi_{n}\left(x_{i}^{\prime}\right)=f\left(x_{i}^{\prime}\right)+\varepsilon_{n} \Psi\left(x_{i}^{\prime}\right)
$$

and

$$
\varphi_{n}\left(x_{i}^{\prime \prime}\right)=f\left(x_{i}^{\prime \prime}\right)+\varepsilon_{n} \Psi\left(x_{i}^{\prime}\right) .
$$

That such a function exists and is uniquely determined has been established by Loewner [6]. See also [5]. It is known that $\varphi_{n}(z)$ is of degree exactly $2 n$ and 
that it has a positive imaginary part in the upper half-plane. It is real on the real axis and has all of its poles there. It is important to notice that these poles are outside the interval $\left(x_{1}^{\prime}, x_{n}^{\prime \prime}\right)$. A well-known theorem guarantees that the sequence $\varphi_{n}(z)$ has a subsequence converging uniformly on compact subsets of the upper half-plane and also uniformly on compact subsets of the interval $\left(a^{\prime}, b^{\prime \prime}\right)$. The limiting function $F(x)$ is manifestly also of the form (1) and coincides with $f(x)$ on the intervals $\left(a^{\prime}, b^{\prime}\right)$ and $\left(a^{\prime \prime}, b^{\prime \prime}\right)$. The associated measure has no mass in the interval $\left(a^{\prime}, b^{\prime \prime}\right)$. Thus $F(x)$ is a monotone operator function when considered on the interval $\left(a^{\prime}, b^{\prime \prime}\right)$. Since the choice of the subintervals $\left(a^{\prime}, b^{\prime}\right)$ and $\left(a^{\prime \prime}, b^{\prime \prime}\right)$ was arbitrary, it is clear that the analytic continuation of $f(x)$ from a constituent interval of $J$ to the half-plane is independent of the choice of the interval, and that the monotone operator function so obtained admits a representation (1) with the associated measure putting no mass in any interval of the form $\left(a_{i}, b_{j}\right)$ where $a_{i}<b_{j}$. This means that $d \mu$ has no mass in the convex hull of $J$.

In the case when $J$ is not bounded, we consider first $f_{N}(x)$, the restriction of $f(x)$ to the intersection of $J$ with the interval $(-N, N)$. It is clear that the analytic continuation of $f_{N}(x)$ to the half-plane is independent of $N$. It is also clear that the measure $d \mu$ associated with that analytic continuation has no mass in the convex hull of the part of $J$ in $(-N, N)$, and since $N$ is arbitrary, there is no mass in $c(J)$, as asserted. This completes the proof.

It is interesting to note that if $c(J)$ is the whole axis then $f(x)$ is necessarily a linear function with nonnegative slope.

A review of the previous proof makes it clear that we made very little use of the hypothesis that $J$ was an open set. Suppose, for example, that $J$ is an arbitrary set such that $c(J)$ is the open interval $(a, b)$, where we do not exclude the possibility of one or more endpoints at infinity. The construction of the sequence $\varphi_{n}(z)$ is still possible, selecting the $x_{k}^{\prime}$ near $a$ and the $x_{k}^{\prime \prime}$ near $b$. The existence of the functions $\varphi_{n}(z)$ depends only on the positivity of certain "Loewner determinants" associated with the points of $S_{n}$ and this is an algebraic, rather than an analytical fact. See [5]. Thus, as in the proof of Chandler's theorem, we find that when $c(J)=(a, b)$ a monotone operator function defined on $J$ is the restriction to that set of a monotone operator function on $c(J)$.

Circumstances are slightly different when $c(J)$ is closed on the left, say $c(J)=[a, b)$. In this case we form the sequence $\varphi_{n}(z)$ as before, only always taking $x_{1}^{\prime}$ as $a$, a point of $J$. The corresponding sequence of Pick functions $\varphi_{n}(z)$ has a convergent subsequence and the limiting function $F(x)$ coincides with the initial $f(x)$ on $J$, except, perhaps, at the point $a$ where the inequality will read $F(a) \geqslant f(a)$.

In a similar way we find that if $c(J)$ is of form $(a, b]$ then $f(x)$ coincides on $J$ with a Pick function throughout the interval, except, perhaps, at the right hand end point where the inequality $F(b) \leqslant f(b)$ is valid. It is also obvious how to treat the case when $c(J)$ is a closed interval. We have therefore established the following result. 
Generalized Chandler Theorem. Let $J$ be an arbitrary subset of the real axis and $c(J)$ its convex hull and let $f(x)$ be a monotone operator function defined on $J$. Then there exists a function $F(x)$ of the form (1) so that $F(x)=f(x)$ at all points of $J$ in the interior of $c(J)$ and satisfying the inequalities $F(z) \geqslant f(z)$ and $F(b) \leqslant f(b)$. The function $F(x)$ is associated with a measure $d \mu$ that puts no mass in $c(J)$.

As a special case of this theorem we obtain a result of Šmul'jan [8]: if $J$ consists of a point $a$ and an interval $(b, c)$ where $a<b$ and $f(x)$ is a monotone operator function on $J$, then $f(x)$ admits an extension $F(x)$ to a moonotone operator function defined on $[a, c)$ which coincides with $f(x)$ on $(b, c)$ and satisfies the inequality $F(a) \geqslant f(a)$.

\section{REFERENCES}

1. J. Bendat and S. Sherman, Monotone and convex operator functions, Trans. Amer. Math. Soc. 79 (1955), 58-71. MR 18, 588.

2. J. D. Chandler, Jr., Extensions of monotone operator functions, Proc. Amer. Math. Soc. 54 (1976), 221-224.

3. C. Davis, A Schwarz inequality for convex operator functions, Proc. Amer. Math. Soc. 8 (1957), 42-44. MR 18, 812.

4. Notions generalizing convexity for functions defined on spaces of matrices, Proc. Sympos. Pure Math., vol. 7, Amer. Math. Soc., Providence, R. I., 1963, pp. 187-201. MR 27 \#5771.

5. W. F. Donoghue, Jr., Monotone matrix functions and analytic continuation, Springer-Verlag, New York and Berlin, 1974.

6. K. Löwner, Über monotone Matrixfunktionen, Math. Z. 38 (1934), 177-216.

7. M. Rosenblum and J. Rovnyak, Restrictions of analytic functions. I, Proc. Amer. Math. Soc. 48 (1975), 113-119.

8. Ju. L. Smul'jan, Monotone operator functions on a set consisting of an interval and a point, Ukrain. Mat. Ž. 17 (1965), no. 1, 130-136; English transl., Amer. Math. Soc. Transl. (2) 67 (1968), 25-32. MR 32 \#6239.

Department of Mathematics, University of California, Irvine, California 92717 